\title{
On two-primary algebraic K-theory of quadratic number rings with focus on $K_{2}$
}

\author{
by \\ Marius Crainic (Utrecht) and Paul Arne Østvær (Oslo)
}

1. Introduction. This paper deals with the two-primary algebraic Ktheory of quadratic number rings. The problem is to determine the torsion part of these groups. In [14] Quillen proved that the algebraic K-groups of number rings are finitely generated Abelian groups, and Borel calculated their rank in [2].

To determine the torsion part of the algebraic K-groups of number rings is a very hard problem. One knows quite a lot about the $K_{2}$-functor, but still it is a highly nontrivial problem to determine the $K_{2}$-groups. A major early contribution to the theory is Tate's work in [16] that gives connections between $K_{2}$ and Galois cohomology. In fact $p$-rank formulas for $K_{2}$ of number rings can be quite easily deduced from results in [16]. These formulas involve classical number theory like ideal class groups and the number of primes lying above a rational prime. Unfortunately $p$ is in general restricted to be a prime number. The situation is a bit better when it comes to quadratic number rings. In that case there are 4-rank formulas for $K_{2}$ given in terms of classical number-theoretic invariants.

In this paper we describe a method to determine pieces of the twoprimary part of $K_{2}$ of a quadratic number ring. There is a tight connection between the ordinary and the narrow $K_{2}$. This connection will be explained in the calculations. To the authors' knowledge, the latest and most complete calculation of $K_{2}$ of quadratic number rings can be found in [12] and [13]. We

1991 Mathematics Subject Classification: 11R11, 11R65, 14F20, 19C99, $19 \mathrm{D} 50$.

Key words and phrases: algebraic K-groups of quadratic number rings, 2- and 4-rank formulas for Picard groups, étale cohomology.

Both authors thank the Mathematical Research Institute, MRI, in The Netherlands, in particular F. Keune. The first author was supported by a research fellowship during the MRI Master Class 1995/1996. Furthermore we thank H. Qin for useful conversations leading to Remark 3.10. Finally we heartily thank J. Rognes and C. Weibel for freely sharing the results in [15] with us, and an anonymous correspondent for a careful reading of the paper. 
would also like to mention the recent papers [17] and [18]. Further a large table of conjectural calculations is given in [3]. Our method can be used to capture the previous calculations as well. Furthermore we lift the $K_{2}$ calculations to higher even algebraic K-groups. This is done using the étale cohomology description of the two-primary algebraic K-theory of number rings from [15].

For the rest of the paper we fix the following notation:

- $F$ is a number field with number ring $\mathcal{O}_{F}$,

- $R_{F}$ denotes the ring of two-integers of $F$,

- $\Delta_{F}$ denotes the discriminant of $F$,

- $s$ is the number of dyadic primes in $F$, i.e., the number of primes lying above 2 in $F$,

- $t$ is the number of distinct prime divisors of $\Delta_{F}$,

- $r_{1}$ is the number of real embeddings of $F$,

- $d$ denotes a squarefree integer,

- Pic denotes the Picard group and Pic + denotes the narrow Picard group,

- $A\{n\}$ denotes the $n$-primary part of the Abelian group $A$ and $\operatorname{rk}_{n}(A)$ the $n$-rank of $A$, where $n$ is a prime power,

- $C_{n}$ is the cyclic group of order $n$.

The following result concerns the algebraic K-theory of $R_{F}$. However, the fibre sequence of spectra

$$
\bigvee_{\wp \mid 2} K\left(k_{\wp}\right) \rightarrow K\left(\mathcal{O}_{F}\right) \rightarrow K\left(R_{F}\right)
$$

implies that the two-primary parts of the algebraic K-groups of $\mathcal{O}_{F}$ and $R_{F}$ are isomorphic from degree 2 on. This is an immediate consequence of the long exact sequence of two-completed algebraic K-groups induced by the fibre sequence above, and Quillen's calculation of algebraic K-theory of finite fields.

We recall Theorem 7.11 of [15]. We alert the reader that $t$ denotes $\operatorname{rk}_{2} \operatorname{Pic}\left(R_{F}\right)$ and $u$ denotes $\operatorname{rk}_{2} \operatorname{Pic}_{+}\left(R_{F}\right)$ in [15]. We make this result explicit for quadratic number fields in Theorem 3.1.

TheOREM 1.1 (Rognes-Weibel). Let $n \geq 1$. The number of even cyclic summands in the algebraic K-groups of $R_{F}$ are given by

(a) If $F$ is totally imaginary, then

$$
\operatorname{rk}_{2} K_{n}\left(R_{F}\right)= \begin{cases}s+\operatorname{rk}_{2} \operatorname{Pic}\left(R_{F}\right)-1 & \text { for } n \text { even }, \\ 1 & \text { for } n \text { odd } .\end{cases}
$$


(b) If $F$ is real, then

$$
\operatorname{rk}_{2} K_{n}\left(R_{F}\right)= \begin{cases}s+\operatorname{rk}_{2} \operatorname{Pic}\left(R_{F}\right)-1 & \text { for } n=8 i, \\ 1 & \text { for } n=8 i+1, \\ r_{1}+s+\operatorname{rk}_{2} \operatorname{Pic}\left(R_{F}\right)-1 & \text { for } n=8 i+2, \\ r_{1} & \text { for } n=8 i+3, \\ s+\operatorname{rk}_{2} \operatorname{Pic}_{+}\left(R_{F}\right)-1 & \text { for } n=8 i+4, \\ 0 & \text { for } n=8 i+5, \\ s+\operatorname{rk}_{2} \operatorname{Pic}_{+}\left(R_{F}\right)-1 & \text { for } n=8 i+6, \\ 1 & \text { for } n=8 i+7 .\end{cases}
$$

Now a quick outline of the paper. Fix $F=\mathbb{Q}(\sqrt{d})$ and $\bar{F}=\mathbb{Q}(\sqrt{-d})$.

In Section 2 we compute $\operatorname{rk}_{2} \mathrm{Pic}\left(R_{F}\right)$ and $\operatorname{rk}_{2} \mathrm{Pic}_{+}\left(R_{F}\right)$. Further we compare $\operatorname{rk}_{4} \mathrm{Pic}_{+}\left(\mathcal{O}_{F}\right)$ and $\operatorname{rk}_{4} \mathrm{Pic}_{+}\left(R_{F}\right)$. Here the number $\operatorname{rk}_{4} \mathrm{Pic}_{+}\left(\mathcal{O}_{F}\right)$ can be computed using a matrix $M_{F}$ described in Subsection 3.2. The number $\mathrm{rk}_{4} \mathrm{Pic}_{+}\left(R_{F}\right)$ occurs in 4-rank formulas for both $K_{2}^{+}\left(R_{F}\right)$ and $K_{2}\left(R_{F}\right)$ given in [1] (see Theorems 3.3 and 3.4 in this paper). From the comparison of $\operatorname{rk}_{4} \mathrm{Pic}_{+}\left(\mathcal{O}_{F}\right)$ and $\mathrm{rk}_{4} \mathrm{Pic}_{+}\left(R_{F}\right)$ we compute in Proposition 3.7 the 4-rank of $K_{2}^{+}\left(R_{F}\right)$ in terms of $\operatorname{rk}_{4} \mathrm{Pic}_{+}\left(\mathcal{O}_{\bar{F}}\right)$. The 4-rank of $K_{2}\left(R_{F}\right)$ can then be found using Theorems 2.9, 3.3 and 3.4.

In Subsection 3.3 we give several examples of two-primary calculations of $K_{2}\left(R_{F}\right)$. In some cases we can completely determine the two-primary part of $K_{2}\left(R_{F}\right)$. To obtain more information on $K_{2}\left(R_{F}\right)\{2\}$ one would need a $2^{\nu}$-rank formula for $K_{2}\left(R_{F}\right)$ where $\nu \geq 3$.

In Subsection 3.4 we use the étale cohomology description of the twoprimary algebraic K-theory of $R_{F}$ given in [15] to lift the $K_{2}$ calculations to higher even algebraic K-groups of $R_{F}$. The main result is

TheOREM 1.2. Let $F$ be any number field. Let $e$ be the exponent of $\left(\mathbb{Z} / 2^{\nu}\right)^{*}$ where $\nu \geq 2$ if $F$ is a real number field. Then

$$
K_{2}\left(R_{F}\right) / 2^{\nu} \cong H_{\text {ét }}^{2}\left(R_{F} ; \mathbb{Z}_{2}(e+2)\right) / 2^{\nu} .
$$

2. 2-rank and 4-rank formulas for quadratic Picard groups. In this section we will give 2-rank formulas for "quadratic Picard groups". These results will be vital when we turn to the two-primary algebraic Ktheory of quadratic number rings. First we set the notation and recall some classical results.

Let $S$ denote a finite set of primes in $F$ including the infinite ones, and $\mathcal{O}_{F, S}$ the ring of $S$-integers in $F$. Let $\mathrm{Pic}_{+}\left(\mathcal{O}_{F, S}\right)$ denote the narrow Picard group of the $S$-integers and $\operatorname{Pic}\left(\mathcal{O}_{F, S}\right)$ the usual Picard group of $\mathcal{O}_{F, S}$. These groups come with a natural surjective map

$$
\iota_{S}: \mathrm{Pic}_{+}\left(\mathcal{O}_{F, S}\right) \rightarrow \operatorname{Pic}\left(\mathcal{O}_{F, S}\right) .
$$


For a fractional ideal $\mathfrak{a}$ in a number field $F$ we let $[\mathfrak{a}]$ denote the class of $\mathfrak{a}$ in $\operatorname{Pic}\left(\mathcal{O}_{F, S}\right)$ while $[\mathfrak{a}]_{+}$denotes the class of $\mathfrak{a}$ in $\operatorname{Pic}_{+}\left(\mathcal{O}_{F, S}\right)$. If $S$ contains no finite primes then we obtain the ordinary and the narrow ideal class group. The notation will be $\operatorname{Pic}\left(\mathcal{O}_{F}\right)$ and $\operatorname{Pic}_{+}\left(\mathcal{O}_{F}\right)$, respectively. In this case we denote $\iota_{S}$ with $\iota$. We mention two classical results.

Lemma 2.1. Let $F$ be a real quadratic number field with fundamental unit $\varepsilon$. Then

$$
\operatorname{ker} \iota \cong \begin{cases}C_{2} & \text { if } \mathbf{N}_{F / \mathbb{Q}}(\varepsilon)=1, \\ 1 & \text { otherwise. }\end{cases}
$$

Pr o of. We refer to [6], p. 181.

For quadratic number fields, the 2-rank of $\mathrm{Pic}_{+}\left(\mathcal{O}_{F}\right)$ was known to Gauss.

Theorem 2.2 (Gauss). Let $F$ be a quadratic number field. Then

$$
\operatorname{rk}_{2} \operatorname{Pic}_{+}\left(\mathcal{O}_{F}\right)=t-1 \text {. }
$$

Proof. A short and elegant proof can be found in [7].

2.1. The 2-rank of the ideal class group of quadratic number fields. As an application of the classical 2-rank formula for $\operatorname{rk}_{2} \mathrm{Pic}_{+}\left(\mathcal{O}_{F}\right)$ we will now find the 2-rank of $\operatorname{Pic}\left(\mathcal{O}_{F}\right)$. First we make some preparations.

DeFinition 2.3. Let $k$ be an integer. We define

$M_{k}:=\{d \in \mathbb{Z} \mid d$ is squarefree and $k$ is a norm from $F=\mathbb{Q}(\sqrt{d})\}$.

LEMMA 2.4. We have:

$M_{-1}=\{d \in \mathbb{Z} \mid d>0, p \equiv 1 \bmod 4$ for every odd prime $p$ dividing $d\}$, $M_{-2}=\{d \in \mathbb{Z} \mid d>0, p \equiv 1$ or $3 \bmod 8$ for every odd prime $p$ dividing $d\}$, $M_{2}=\{d \in \mathbb{Z} \mid p \equiv 1$ or $7 \bmod 8$ for every odd prime $p$ dividing $d\}$.

Proof. For basic properties of Hilbert symbols we refer to [11]. The product formula for Hilbert symbols tells us that

$$
\prod_{\wp}(k, d)_{\wp}=1
$$

where $\wp$ runs over the finite and infinite primes of $\mathbb{Q}$, and $k$ is an integer as in Definition 2.3. The celebrated Hasse norm theorem in [11], p. 89, reads as

$$
d \in M_{k} \Leftrightarrow(k, d)_{\wp}=1 \text { for all primes } \wp \text { of } \mathbb{Q} \text {. }
$$

Hence from the product formula, $d \in M_{k}$ only if $(k, d)_{\wp}=1$ for all finite and infinite primes $\wp$ of $\mathbb{Q}$ except 2 . Let $\nu_{\infty}$ be the infinite prime in $\mathbb{Q}$. Then $(k, d)_{\nu_{\infty}}$ equals -1 if and only if both $k$ and $d$ are negative. Furthermore for 
an odd rational prime $p$,

$$
(k, d)_{p}= \begin{cases}\left(\frac{k}{p}\right) & \text { if } p \mid d \text { and } p \nmid k, \\ \left(\frac{d}{p}\right) & \text { if } p \nmid d \text { and } p \mid k, \\ \left(\frac{-k d / p^{2}}{p}\right) & \text { if } p \mid d \text { and } p \mid k, \\ 1 & \text { if } p \nmid d \text { and } p \nmid k .\end{cases}
$$

Thus if $k$ has no odd prime divisors (e.g., $k=-1,-2$ or 2 ), then

$$
d \in M_{k} \Leftrightarrow(k>0 \text { or } d>0) \text { and }\left(\left(\frac{k}{p}\right)=1 \text { for all odd primes } p \text { dividing } d\right) \text {. }
$$

We easily read off the result from the well known formulas $\left(\frac{-1}{p}\right)=$ $(-1)^{(p-1) / 2}$ and $\left(\frac{2}{p}\right)=(-1)^{\left(p^{2}-1\right) / 8}$.

We will need the following elementary group-theoretic result.

Lemma 2.5. For a finite Abelian group $G$ and $x \in G$,

$$
\operatorname{rk}_{2}(G /\langle x\rangle)= \begin{cases}\operatorname{rk}_{2}(G) & \text { if } x \in G^{2} \\ \operatorname{rk}_{2}(G)-1 & \text { otherwise. }\end{cases}
$$

Pro of. Say $x$ generates a subgroup of order $n,\langle x\rangle=C_{n}$. Further let $G^{\prime}$ denote the quotient group, i.e., we have the exact sequence $0 \rightarrow C_{n} \rightarrow G \rightarrow$ $G^{\prime} \rightarrow 0$ inducing the exact sequence $C_{n} / 2 \rightarrow G / 2 \rightarrow G^{\prime} / 2 \rightarrow 0$.

There are two cases to consider.

1) Suppose that $2 \nmid n$. Now $C_{n}^{2}=C_{n}$, and hence $C_{n} / 2=0$. Thus $x \in G^{2}$ and $\operatorname{rk}_{2}(G)=\operatorname{rk}_{2}\left(G^{\prime}\right)$.

2) Otherwise $2 \mid n$. Now $C_{n} / 2=C_{2}$ and the sequence $C_{2} \stackrel{\theta}{\rightarrow} G / 2 \rightarrow$ $G^{\prime} / 2 \rightarrow 0$ is exact. If $x \in G^{2}$, then $\theta$ must be trivial and $\operatorname{rk}_{2}(G)=\operatorname{rk}_{2}\left(G^{\prime}\right)$. If $x \notin G^{2}$, then $\theta$ is injective. Hence $0 \rightarrow C_{2} \rightarrow G / 2 \rightarrow G^{\prime} / 2 \rightarrow 0$ is exact, and $\sharp(G / 2)=2 \sharp\left(G^{\prime} / 2\right)$.

The next lemma will be used several times. To avoid possible confusion we adopt the notation that $\mathbf{N}$ denotes the ideal norm, while $\mathbf{N}_{F / \mathbb{Q}}$ denotes the element norm from $F$ to $\mathbb{Q}$. For the connection between them, see p. 57 of [6]. First we note the following easy observation: a norm 1 fractional ideal can be written in the form $\mathfrak{c} / \overline{\mathfrak{c}}$ where $\overline{\mathfrak{c}}$ denotes the Galois conjugate of $\mathfrak{c}$. Here $\mathfrak{c}$ equals a product of primes lying above split rational primes in $\mathbb{Q}$. It is easy to prove this. Start with a norm 1 fractional ideal, say $\mathfrak{a}$. Write $\mathfrak{a}=\mathfrak{b} / \mathfrak{c}$ where $\mathfrak{b}$ and $\mathfrak{c}$ are coprime integral $\mathcal{O}_{F}$-ideals. If $\wp$ is a prime ideal that divides $\mathfrak{b}$, then there must exist a prime ideal $\wp$ that divides $\mathfrak{c}$ and with the same norm as $\wp$. Since $(\mathfrak{b}, \mathfrak{c})=1$ we know that $\wp \neq \varnothing$. Hence working in a quadratic number field we conclude that $\wp$ and $\bar{\wp}$ are conjugate. 
Lemma 2.6. For a fractional ideal $\mathfrak{a}$ of $F=\mathbb{Q}(\sqrt{d})$ the following hold:

$$
\begin{aligned}
{[\mathfrak{a}] \in \operatorname{Pic}\left(\mathcal{O}_{F}\right)^{2} } & \Leftrightarrow \mathbf{N}(\mathfrak{a}) \text { or }-\mathbf{N}(\mathfrak{a}) \text { is a norm from } F, \\
{[\mathfrak{a}]_{+} \in \operatorname{Pic}_{+}\left(\mathcal{O}_{F}\right)^{2} } & \Leftrightarrow \mathbf{N}(\mathfrak{a}) \text { is a norm from } F^{+} .
\end{aligned}
$$

Pr o of. $\Rightarrow$ : Suppose that $[\mathfrak{a}] \in \operatorname{Pic}\left(\mathcal{O}_{F}\right)^{2}$, respectively $[\mathfrak{a}]_{+} \in \operatorname{Pic}_{+}\left(\mathcal{O}_{F}\right)^{2}$. Then we can find $\alpha \in F^{*}$, respectively $\alpha \in F^{+}$, and a fractional ideal $\mathfrak{b}$ such that $\mathfrak{a}=(\alpha) \mathfrak{b}^{2}$. We derive

$$
\left|\mathbf{N}_{F / \mathbb{Q}}(\alpha \mathbf{N}(\mathfrak{b}))\right|=\left|\mathbf{N}_{F / \mathbb{Q}}(\alpha)\right| \mathbf{N}\left(\mathfrak{b}^{2}\right)=\mathbf{N}\left((\alpha) \mathfrak{b}^{2}\right)=\mathbf{N}(\mathfrak{a}) .
$$

For the narrow case we have $\mathbf{N}_{F / \mathbb{Q}}(\alpha \mathbf{N}(\mathfrak{b}))>0$, so we cannot have a minus sign.

$\Leftarrow$ : Say $\beta \in F^{*}$, respectively $\beta \in F^{+}$, with $\mathbf{N}_{F / \mathbb{Q}}(\beta)= \pm \mathbf{N}(\mathfrak{a})$ (no minus sign in the narrow case). Consider the fractional ideal $\mathfrak{b}=(\beta)^{-1} \mathfrak{a}$. Clearly $\mathfrak{a}$ and $\mathfrak{b}$ represent the same class in both the narrow and the usual ideal class group. Furthermore

$$
\mathbf{N}(\mathfrak{b})=\frac{\mathbf{N}(\mathfrak{a})}{\left|\mathbf{N}_{F / \mathbb{Q}}(\beta)\right|}=1 .
$$

We know that a norm 1 fractional ideal can be written in the form $\mathfrak{d} / \overline{\mathfrak{d}}$ where $\overline{\mathfrak{d}}$ denotes the Galois conjugate of $\mathfrak{d}$. Further $\mathfrak{d} \overline{\mathfrak{d}}$ is a principal fractional ideal representing the trivial class in both the narrow and the usual ideal class group. It follows that

$$
[\mathfrak{a}]=[\mathfrak{b}]=\left[\frac{\mathfrak{d}^{2}}{\mathfrak{d} \overline{\mathfrak{d}}}\right]=[\mathfrak{d}]^{2} .
$$

Of course the same holds for the narrow classes as well.

Now we have all the results we need in order to find $\operatorname{rk}_{2} \operatorname{Pic}\left(\mathcal{O}_{F}\right)$.

Theorem 2.7. For $F=\mathbb{Q}(\sqrt{d})$ we have $\operatorname{rk}_{2} \operatorname{Pic}\left(\mathcal{O}_{F}\right)= \begin{cases}t-1 & \text { if } d<0 \text { or } \\ & (d>0 \text { and } p \equiv 1 \bmod 4 \text { for all odd primes } p \mid d), \\ t-2 & \text { if } d>0 \text { and } p \equiv 3 \bmod 4 \text { for some odd prime } p \mid d .\end{cases}$

Proof. We may assume $d>0$, and due to Lemma 2.4 it suffices to show that

$$
\operatorname{rk}_{2} \operatorname{Pic}\left(\mathcal{O}_{F}\right)= \begin{cases}t-1 & \text { if }-1 \text { is a norm from } F, \\ t-2 & \text { otherwise. }\end{cases}
$$

The sequence $0 \rightarrow \operatorname{ker} \iota \rightarrow \operatorname{Pic}_{+}\left(\mathcal{O}_{F}\right) \rightarrow \operatorname{Pic}\left(\mathcal{O}_{F}\right) \rightarrow 0$ is exact, where ker $\iota$ is known from Lemma 2.1. This gives us the exact sequence ker $\iota / 2 \rightarrow$ $\operatorname{Pic}_{+}\left(\mathcal{O}_{F}\right) / 2 \rightarrow \operatorname{Pic}\left(\mathcal{O}_{F}\right) / 2 \rightarrow 0$. Clearly ker $\iota$ is generated by some $[(a)]_{+}$ where $a$ has negative norm. From Lemma 2.5 we are reduced to proving that

$$
[(a)]_{+} \in \operatorname{Pic}_{+}\left(\mathcal{O}_{F}\right)^{2} \Leftrightarrow d \in M_{-1}
$$

for any element $a \in F^{*}$ with negative norm. 


$$
\begin{aligned}
\Rightarrow & \text { : Say }[(a)]_{+}=[\mathfrak{a}]_{+}^{2}, \text { i.e., }(a)=(\alpha) \mathfrak{a}^{2} \text { for some } \alpha \in F^{+} \text {. We get } \\
& \mathbf{N}((a))=\mathbf{N}((\alpha)) \mathbf{N}(\mathfrak{a})^{2}, \quad \text { i.e., } \quad-\mathbf{N}_{F / \mathbb{Q}}(a)=\mathbf{N}_{F / \mathbb{Q}}(\alpha) \mathbf{N}(\mathfrak{a})^{2}
\end{aligned}
$$

and hence

$$
-1=\mathbf{N}_{F / \mathbb{Q}}\left(\frac{\alpha}{a} \mathbf{N}(\mathfrak{a})\right) \in \mathbf{N}_{F / \mathbb{Q}}(F) .
$$

$\Leftarrow$ : Choose $\alpha \in F^{*}$ where $\mathbf{N}_{F / \mathbb{Q}}(\alpha)=-1$, i.e., $\mathbf{N}((\alpha))=1$. The rest of the argument can be found in the proof of Lemma 2.6.

REMARK 2.8. The short exact sequence

$$
0 \rightarrow \operatorname{ker} \iota \rightarrow \operatorname{Pic}_{+}\left(\mathcal{O}_{F}\right) \stackrel{\iota}{\rightarrow} \operatorname{Pic}\left(\mathcal{O}_{F}\right) \rightarrow 0
$$

is not in general split exact. One example is $F=\mathbb{Q}(\sqrt{34})$. In this case $\mathbf{N}_{F / \mathbb{Q}}(\varepsilon)=1$ where $\varepsilon=35+6 \sqrt{34}$ denotes the fundamental unit. Hence ker $\iota \cong C_{2}$. From Theorem 2.7 we see that

$$
\operatorname{rk}_{2} \operatorname{Pic}\left(\mathcal{O}_{\mathbb{Q}(\sqrt{34})}\right)=\operatorname{rk}_{2} \operatorname{Pic}_{+}\left(\mathcal{O}_{\mathbb{Q}(\sqrt{34})}\right)=1 .
$$

In fact the ordinary ideal class group is cyclic of order 2 , so the narrow ideal class group is cyclic of order 4 .

2.2. The 2-rank of $\mathrm{Pic}$ and $\mathrm{Pic}_{+}$for the two-integers in quadratic number fields. We will now see what happens with the 2 -rank of the narrow and the usual Picard group of the two-integers of $F=\mathbb{Q}(\sqrt{d})$.

Theorem 2.9. For $F=\mathbb{Q}(\sqrt{d})$ we have

$$
\begin{aligned}
\operatorname{rk}_{2} \operatorname{Pic}\left(R_{F}\right) & = \begin{cases}\operatorname{rk}_{2} \operatorname{Pic}\left(\mathcal{O}_{F}\right) & \text { if } d \equiv 5 \bmod 8 \text { or } d \in M_{-2} \cup M_{2}, \\
\operatorname{rk}_{2} \operatorname{Pic}\left(\mathcal{O}_{F}\right)-1 & \text { otherwise, }\end{cases} \\
\operatorname{rk}_{2} \operatorname{Pic}_{+}\left(R_{F}\right) & = \begin{cases}\operatorname{rk}_{2} \operatorname{Pic}_{+}\left(\mathcal{O}_{F}\right) & \text { if } d \equiv 5 \bmod 8 \text { or } d \in M_{2}, \\
\operatorname{rk}_{2} \operatorname{Pic}_{+}\left(\mathcal{O}_{F}\right)-1 & \text { otherwise. }\end{cases}
\end{aligned}
$$

Proof. The crucial thing here is of course the factorization of 2 in $F$. There are three possibilities:

1) If $d \equiv 1 \bmod 8$, then 2 splits,

2) If $d \equiv 3 \bmod 4$ or $2 \mid d$, then 2 ramifies,

3 ) If $d \equiv 5 \bmod 8$, then 2 is inert.

In the cases where 2 is inert, i.e., when $d \equiv 5 \bmod 8$ there will be no effect on neither the narrow nor the ordinary ideal class group if we invert 2 . Hence we may assume that the residue field index of 2 is not two, so $\mathbf{N}(\wp)=2$ for $\wp$ a dyadic prime. Clearly $\operatorname{Pic}\left(R_{F}\right)=\operatorname{Pic}\left(\mathcal{O}_{F}\right) /\langle[\wp]\rangle$ for $\wp$ a dyadic prime of $F$. The situation for the narrow ideal class group is identical, i.e., $\operatorname{Pic}_{+}\left(R_{F}\right)=\operatorname{Pic}_{+}\left(\mathcal{O}_{F}\right) /\left\langle[\wp]_{+}\right\rangle$. We are left with proving that

$[\wp] \in \operatorname{Pic}\left(\mathcal{O}_{F}\right)^{2} \Leftrightarrow \pm 2 \in \mathbf{N}_{F / \mathbb{Q}}(F)$ (only the plus sign in the narrow case).

Now mimic the proof of Lemma 2.6 and Theorem 2.7. 
Later we will need the following explicit description of $\operatorname{rk}_{2} \operatorname{Pic}\left(R_{F}\right)$.

COROLlary 2.10. For $F=\mathbb{Q}(\sqrt{d})$ we have the following calculation.

1) $\operatorname{rk}_{2} \operatorname{Pic}\left(R_{F}\right)=t-1$ in the cases:

- $d<0, d \equiv 5 \bmod 8$,

- $d<0, p \equiv 1$ or $7 \bmod 8$ for every odd prime $p$ dividing $d$,

- $d>0, d \equiv 5 \bmod 8$ and $p \equiv 1 \bmod 4$ for every odd prime $p$ dividing $d$,

- $d>0, p \equiv 1 \bmod 8$ for every odd prime $p$ dividing $d$.

2) $\operatorname{rk}_{2} \operatorname{Pic}\left(R_{F}\right)=t-3$ in the case

- $d>0, d \equiv 1,2,3,6$ or $7 \bmod 8$ and there exist prime numbers $p_{1}$ and $p_{2}$ dividing $d$ where $p_{1} \equiv 5 \bmod 8$ and $p_{2} \equiv 3 \bmod 4$.

3) $\operatorname{rk}_{2} \operatorname{Pic}\left(R_{F}\right)=t-2$ in all other cases.

Pr o of. We compare Theorems 2.7 and 2.9. There are two cases to consider.

1) The case $\operatorname{rk}_{2} \operatorname{Pic}\left(R_{F}\right)=\operatorname{rk}_{2} \operatorname{Pic}\left(\mathcal{O}_{F}\right)=t-1$. If $F$ is imaginary, then $d \equiv 5 \bmod 8$ or $d \in M_{2}$. Lemma 2.4 gives the claimed restriction on $d$. For the real case we have $d \equiv 5 \bmod 8$ or $d \in M_{-2} \cup M_{2}$, while $p \equiv 1 \bmod 4$ for every odd prime $p$ dividing $d$. If $d \in M_{-2}$, then $p \equiv 1$ or $3 \bmod 8$ for every odd prime $p$ dividing $d$. If $d \in M_{2}$, then $p \equiv 1$ or $7 \bmod 8$ for every odd prime $p$ dividing $d$.

2) The case $\operatorname{rk}_{2} \operatorname{Pic}\left(R_{F}\right)=t-3$. From the formula for $\operatorname{rk}_{2} \operatorname{Pic}\left(\mathcal{O}_{F}\right), d$ is positive and $d \notin M_{-1}$, i.e., there exists an odd prime congruent to 3 modulo 4 and dividing $d$. The "otherwise" condition in the formula for $\operatorname{rk}_{2} \operatorname{Pic}\left(R_{F}\right)$ is that $d \equiv 1,2,3,6$ or $7 \bmod 8$ and that there exists an odd prime dividing $d$ and congruent to 5 modulo 8 .

A straightforward comparison of Theorems 2.2 and 2.9 yields

COROLLARY 2.11. We have the following formula for the 2-rank of the narrow Picard group of the 2-integers in $F=\mathbb{Q}(\sqrt{d})$ :

$$
\mathrm{rk}_{2} \mathrm{Pic}_{+}\left(R_{F}\right)= \begin{cases}t-1 & \text { if } d \equiv 5 \bmod 8 \text { or } d \in M_{2} \\ t-2 & \text { otherwise. }\end{cases}
$$

We also note the following two results.

Corollary 2.12. Let $F=\mathbb{Q}(\sqrt{d})$. We have $\operatorname{rk}_{2} \operatorname{Pic}\left(R_{F}\right)=0$ in precisely the following cases: 


\begin{tabular}{cc}
\hline$d$ & with the condition \\
\hline$-1, \pm 2$ & $p \equiv 3 \bmod 4$ or $p \equiv 5 \bmod 8$ \\
$-p$ & $p \equiv 3 \bmod 8$ or $p \equiv 5 \bmod 8$ \\
$-2 p$ & $p_{1} \equiv 3 \bmod 8$ and $p_{2} \equiv 5 \bmod 8$ \\
$-p_{1} p_{2}$ & $p$ is odd \\
$p$ & $p \equiv 3 \bmod 4$ or $p \equiv 5 \bmod 8$ \\
$2 p$ & $p_{1} \equiv 5 \bmod 8$ and $p_{2} \equiv 3 \bmod 4$ \\
$p_{1} p_{2}$ & $p_{1} \equiv 5 \bmod 8$ and $p_{2} \equiv 3 \bmod 4$ \\
$2 p_{1} p_{2}$ & $p_{1} \equiv 3 \bmod 4$ and $p_{2} \equiv 3 \bmod 4$ \\
$p_{1} p_{2}$ & $p_{1} \equiv 5 \bmod 8$ and $p_{2} \equiv 5 \bmod 8$ \\
$p_{1} p_{2}$ & $p_{1} \equiv 5 \bmod 8$ and $p_{2}, p_{3} \equiv 3 \bmod 4$ \\
$p_{1} p_{2} p_{3}$ &
\end{tabular}

P r o of. Consult Corollary 2.10 where we calculated $\operatorname{rk}_{2} \operatorname{Pic}\left(R_{F}\right)$. We omit the details.

Corollary 2.13. Let $F=\mathbb{Q}(\sqrt{d})$ with d positive. We have $\operatorname{rk}_{2} \operatorname{Pic}_{+}\left(R_{F}\right)$ $=0$ in precisely the following cases:

\begin{tabular}{cc}
\hline$d$ & with the condition \\
\hline 2 & $p \equiv 1 \bmod 4$ \\
$p$ & $p \equiv 3 \bmod 8$ or $p \equiv 5 \bmod 8$ \\
$2 p$ & $p_{1} \equiv 3 \bmod 8$ and $p_{2} \equiv 3 \bmod 8$ \\
$p_{1} p_{2}$ & $p_{1} \equiv 5 \bmod 8$ and $p_{2} \equiv 5 \bmod 8$ \\
$p_{1} p_{2}$ & $p_{1}$ \\
\hline
\end{tabular}

P r o of. Use Corollary 2.11.

2.3. The 4-rank of $\mathrm{Pic}_{+}\left(\mathcal{O}_{F}\right)$ and $\mathrm{Pic}_{+}\left(R_{F}\right)$ for $F$ a quadratic number field. Next we would like to compare the 4-rank of $\operatorname{Pic}_{+}\left(\mathcal{O}_{F}\right)$ with the 4-rank of $\mathrm{Pic}_{+}\left(R_{F}\right)$. This turns out to be harder than for the 2-rank. We start with a result about the 4-rank of a finite Abelian group. For an element $x$ in a group $G$, let $\operatorname{ord}(x)$ denote the order of the subgroup generated by $x$.

Lemma 2.14. For a finite Abelian group $G$ and $x \in G$,

$\operatorname{rk}_{4}(G /\langle x\rangle)= \begin{cases}\operatorname{rk}_{4}(G) & \text { if } x \in G^{4}, \text { or }\left(x \notin G^{2} \text { and } \operatorname{ord}(x) \equiv 2 \bmod 4\right) \\ \operatorname{rk}_{4}(G)-1 & \text { or }\left(x \notin G^{2} \text { and } x^{2} \in G^{4} \text { and } \operatorname{ord}(x) \equiv 0 \bmod 4\right),\end{cases}$

Proof. This goes like the analogous lemma we had for the 2-rank. We use the same notion, namely that $x$ generates a subgroup of order $n,\langle x\rangle=C_{n}$. Further let $G^{\prime}$ denote the quotient group. The exact sequence $0 \rightarrow C_{n} \rightarrow$ $G \rightarrow G^{\prime} \rightarrow 0$ induces the exact sequences

$$
C_{n} / 2 \stackrel{\theta}{\rightarrow} G / 2 \rightarrow G^{\prime} / 2 \rightarrow 0 \quad \text { and } \quad C_{n} / 4 \stackrel{\lambda}{\rightarrow} G / 4 \rightarrow G^{\prime} / 4 \rightarrow 0 .
$$

We divide the proof into the following cases. 
1) Suppose $x \in G^{4}$. Then $\operatorname{im}(\lambda)=0$, i.e., $G / 4 \cong G^{\prime} / 4$. In particular their 4-ranks agree. above.

2) Clearly $2 \nmid n$ gives $C_{n}^{4}=C_{n}$ and $x \in C_{n}^{4}$. Hence we are in the situation

3) The case $n \equiv 2 \bmod 4$ gives $C_{n}^{2}=C_{n}^{4}=C_{n / 2}$, so we get the exact sequences

$$
C_{2} \stackrel{\theta}{\rightarrow} G / 2 \rightarrow G^{\prime} / 2 \rightarrow 0 \quad \text { and } \quad C_{2} \stackrel{\lambda}{\rightarrow} G / 4 \rightarrow G^{\prime} / 4 \rightarrow 0 .
$$

- If $x \in G^{2} \backslash G^{4}$, then $\theta$ is trivial and $\lambda$ is injective. Thus $\sharp(G / 2)=\sharp\left(G^{\prime} / 2\right)$ and $\sharp(G / 4)=2 \sharp\left(G^{\prime} / 4\right)$. Therefore $\operatorname{rk}_{2}(G)=\operatorname{rk}_{2}\left(G^{\prime}\right)$ and $\operatorname{rk}_{4}(G)=\operatorname{rk}_{4}\left(G^{\prime}\right)+1$.

- Now for the possibility $x \notin G^{2}$. Both $\theta$ and $\lambda$ are now injective, so $\sharp(G / 2)=2 \sharp\left(G^{\prime} / 2\right)$ and $\sharp(G / 4)=2 \sharp\left(G^{\prime} / 4\right)$. Consequently $\operatorname{rk}_{2}(G)=$ $\operatorname{rk}_{2}\left(G^{\prime}\right)+1$ and $\operatorname{rk}_{4}(G)=\operatorname{rk}_{4}\left(G^{\prime}\right)$.

4) The case $n \equiv 0 \bmod 4$ gives $C_{n}^{2}=C_{n / 2}$ and $C_{n}^{4}=C_{n / 4}$. Hence we get the exact sequences

$$
C_{2} \stackrel{\theta}{\rightarrow} G / 2 \rightarrow G^{\prime} / 2 \rightarrow 0 \quad \text { and } \quad C_{4} \stackrel{\lambda}{\rightarrow} G / 4 \rightarrow G^{\prime} / 4 \rightarrow 0 .
$$

- If $x \in G^{2} \backslash G^{4}$, the map $\theta$ must be trivial while ker $\lambda \cong C_{2}$. As before $\sharp(G / 2)=\sharp\left(G^{\prime} / 2\right)$ and $\sharp(G / 4)=2 \sharp\left(G^{\prime} / 4\right)$. We get $\operatorname{rk}_{2}(G)=\operatorname{rk}_{2}\left(G^{\prime}\right)$ and $\operatorname{rk}_{4}(G)=\operatorname{rk}_{4}\left(G^{\prime}\right)+1$.

- If $x \notin G^{2}$ and $x^{2} \in G^{4}$ then $\theta$ is injective while ker $\lambda \cong C_{2}$. Hence $\sharp(G / 2)=2 \sharp\left(G^{\prime} / 2\right)$ and $\sharp(G / 4)=2 \sharp\left(G^{\prime} / 4\right)$. Thus $\operatorname{rk}_{2}(G)=\operatorname{rk}_{2}\left(G^{\prime}\right)+1$ and $\mathrm{rk}_{4}(G)=\operatorname{rk}_{4}\left(G^{\prime}\right)$.

- The only possibility left is $x \notin G^{2}$ and $x \notin G^{4}$. Now both $\theta$ and $\lambda$ are injective. We get $\sharp(G / 2)=2 \sharp\left(G^{\prime} / 2\right)$ and $\sharp(G / 4)=4 \sharp\left(G^{\prime} / 4\right)$. This implies $\operatorname{rk}_{2}(G)=\operatorname{rk}_{2}\left(G^{\prime}\right)+1$ and $\operatorname{rk}_{4}(G)=\operatorname{rk}_{4}\left(G^{\prime}\right)+1$.

Let $\wp$ be a dyadic prime in $F$. From Lemma 2.14 we know when equality occurs between $\operatorname{rk}_{4} \mathrm{Pic}_{+}\left(\mathcal{O}_{F}\right)$ and $\operatorname{rk}_{4} \mathrm{Pic}_{+}\left(R_{F}\right)$, namely if and only if we are in one of the following cases:

(1) $[\wp]_{+} \in \operatorname{Pic}_{+}\left(\mathcal{O}_{F}\right)^{4}$,

(2) $[\wp]_{+} \notin \operatorname{Pic}_{+}\left(\mathcal{O}_{F}\right)^{2}$ and $\operatorname{ord}\left([\wp]_{+}\right) \equiv 2 \bmod 4$,

(3) $[\wp]_{+} \notin \mathrm{Pic}_{+}\left(\mathcal{O}_{F}\right)^{2},[\wp]_{+}^{2} \in \mathrm{Pic}_{+}\left(\mathcal{O}_{F}\right)^{4}$ and $\operatorname{ord}\left([\wp]_{+}\right) \equiv 0 \bmod 4$.

Otherwise we have $\operatorname{rk}_{4} \mathrm{Pic}_{+}\left(R_{F}\right)=\operatorname{rk}_{4} \mathrm{Pic}_{+}\left(\mathcal{O}_{F}\right)-1$. It is not easy to tell if a class is a fourth power in $\operatorname{Pic}_{+}\left(\mathcal{O}_{F}\right)$. However, we can find answers in some cases.

Lemma 2.15. Let $F=\mathbb{Q}(\sqrt{d})$ where $d \equiv 3$ or $5 \bmod 8$. Then

$$
\mathrm{rk}_{4} \mathrm{Pic}_{+}\left(\mathcal{O}_{F}\right)=\operatorname{rk}_{4} \mathrm{Pic}_{+}\left(R_{F}\right) .
$$

Proof. We know that 2 is inert if $d \equiv 5 \bmod 8$, so we can focus on $d \equiv 3 \bmod 8$. In this case, 2 ramifies and hence $[\wp]_{+}$is trivial or represents 
a class of order two. Recall that $\mathbf{N}(\wp)=2$ in this case. If the order of $[\wp]_{+}$ is 1 , then we are in case (1). If the order is two, we are in case (2) since

$$
[\wp]_{+} \in \operatorname{Pic}_{+}\left(\mathcal{O}_{F}\right)^{2} \Leftrightarrow d \in M_{2} \Rightarrow d \equiv 1,2,6 \text { or } 7 \bmod 8 .
$$

We have already seen proofs of both the equivalence and the implication above, so we will not repeat the argument once more.

Lemma 2.16. For $F=\mathbb{Q}(\sqrt{d})$ where $d \equiv 2,6$ or $7 \bmod 8$ we have $\mathrm{rk}_{4} \mathrm{Pic}_{+}\left(\mathcal{O}_{F}\right)=\mathrm{rk}_{4} \mathrm{Pic}_{+}\left(R_{F}\right)$ if and only if:

(i) $d \notin M_{2}$ or (ii) $[\wp]_{+}$is a fourth power in $\mathrm{Pic}_{+}\left(\mathcal{O}_{F}\right)$.

Pr o of. If (ii) holds, then we are in situation (1). If (i) holds, i.e., $[\wp]_{+} \notin$ $\operatorname{Pic}_{+}\left(\mathcal{O}_{F}\right)^{2}$, then $\operatorname{ord}\left([\wp]_{+}\right)=1$ or ord $\left([\wp]_{+}\right)=2$. These possibilities are covered by (1), respectively (2). Conversely suppose that equality holds. We can be in case (1), which gives us that (ii) is true. Case (2) implies that (i) is true. We can never be in situation (3) since 2 ramifies, and hence the order of $[\wp]_{+}$equals 1 or 2 .

Lemma 2.17. For $F=\mathbb{Q}(\sqrt{d})$ where $d \equiv 1 \bmod 8$ we have $\operatorname{rk}_{4} \operatorname{Pic}_{+}\left(\mathcal{O}_{F}\right)=$ $\mathrm{rk}_{4} \mathrm{Pic}_{+}\left(R_{F}\right)$ if and only if either

1) $[\wp]_{+}$is a fourth power in $\mathrm{Pic}_{+}\left(\mathcal{O}_{F}\right)$, or

$2) \operatorname{ord}\left([\wp]_{+}\right) \equiv 2 \bmod 4$ and $d \notin M_{2}$, or

$3) \operatorname{ord}\left([\wp]_{+}\right) \equiv 0 \bmod 4, d \notin M_{2}$ and $\left([\wp]_{+}^{2}\right) \in \operatorname{Pic}_{+}\left(\mathcal{O}_{F}\right)^{4}$.

Proof. In this case the norm of $\wp$ equals 2 . This is where the condition $d \notin M_{2}$ comes in and replaces $[\wp]_{+}^{2} \notin \operatorname{Pic}_{+}\left(\mathcal{O}_{F}\right)^{2}$.

3. Calculations of two-primary algebraic K-theory for quadratic number rings. In this section we will do the announced algebraic K-theory calculations. From the results on the quadratic Picard groups in Section 2 we can give a complete description of the 2-rank of $K_{n}\left(R_{F}\right)$ for $F$ a quadratic number field. This will be carried out in Subsection 3.1. Further Subsection 3.2 describes a method to determine parts of the two-primary algebraic K-theory of quadratic number rings. Several examples will be treated in Subsection 3.3. The final effort is to lift the $K_{2}$ calculations to higher even algebraic K-groups. This will be done in Subsection 3.4.

3.1. Calculation of $\operatorname{rk}_{2} K_{n}\left(R_{F}\right)$ for $F$ a quadratic number field. We will give a version of Theorem 1.1 for quadratic number fields. Our 2-rank calculation of $\operatorname{Pic}\left(R_{F}\right)$ and $\operatorname{Pic}_{+}\left(R_{F}\right)$ for $F=\mathbb{Q}(\sqrt{d})$ gives an answer in terms of the number of distinct prime divisors $t$ of $\Delta_{F}$ and the number of dyadic primes $s$ in $F$. The integer $s$ is known to be 2 for $d \equiv 1 \bmod 8$ and 1 otherwise.

TheOREM 3.1. Let $F=\mathbb{Q}(\sqrt{d})$ with $d$ squarefree. Let $s$ be the number of dyadic primes and the number of distinct prime divisors of $\Delta_{F}$. We have the following calculation of $\operatorname{rk}_{2} K_{n}\left(R_{F}\right)$ : 
1) Let $F$ be imaginary. If $(d \equiv 5 \bmod 8)$ or $(p \equiv 1$ or $7 \bmod 8$ for all odd primes $p$ dividing $d)$, then:

\begin{tabular}{cc}
\hline$n \geq 1 \bmod 2$ & $\mathrm{rk}_{2} K_{n}\left(R_{F}\right)$ \\
\hline 1 & 1 \\
2 & $s+t-2$ \\
\hline
\end{tabular}

In all other cases we have:

\begin{tabular}{cc}
\hline$n \geq 1 \bmod 2$ & $\mathrm{rk}_{2} K_{n}\left(R_{F}\right)$ \\
\hline 1 & 1 \\
2 & $s+t-3$ \\
\hline
\end{tabular}

2) Let $F$ be real. If $(d \equiv 5 \bmod 8$ and $p \equiv 1 \bmod 4$ for all odd primes $p$ dividing $d)$ or $(p \equiv 1 \bmod 8$ for all odd primes $p$ dividing $d)$, then:

\begin{tabular}{cc}
\hline$n \geq 1 \bmod 8$ & $\mathrm{rk}_{2} K_{n}\left(R_{F}\right)$ \\
\hline 1 & 1 \\
2 & $s+t$ \\
3 & 2 \\
8 & $s+t-2$ \\
\hline
\end{tabular}

If $d \equiv 1,2,3,6$ or $7 \bmod 8$ and there exist odd prime numbers $p_{1}$ and $p_{2}$ dividing $d$ such that $p_{1} \equiv 5 \bmod 8$ and $p_{2} \equiv 3 \bmod 4$, then:

\begin{tabular}{cc}
\hline$n \geq 1 \bmod 8$ & $\mathrm{rk}_{2} K_{n}\left(R_{F}\right)$ \\
\hline 1 & 1 \\
2 & $s+t-2$ \\
3 & 2 \\
8 & $s+t-4$ \\
\hline
\end{tabular}

In all other cases we have:

\begin{tabular}{cc}
\hline$n \geq 1 \bmod 8$ & $\mathrm{rk}_{2} K_{n}\left(R_{F}\right)$ \\
\hline 1 & 1 \\
2 & $s+t-1$ \\
3 & 2 \\
8 & $s+t-3$ \\
\hline
\end{tabular}

For the other values of $n$ modulo 8 we have the following. If ( $d \equiv 5 \bmod 8)$ or $(p \equiv 1$ or $7 \bmod 8)$ for all odd primes $p$ dividing $d$, then:

\begin{tabular}{cc}
\hline$n \geq 1 \bmod 8$ & $\mathrm{rk}_{2} K_{n}\left(R_{F}\right)$ \\
\hline 4 & $s+t-2$ \\
5 & 0 \\
6 & $s+t-2$ \\
7 & 1 \\
\hline
\end{tabular}


In all other cases:

\begin{tabular}{cc}
\hline$n \geq 1 \bmod 8$ & $\mathrm{rk}_{2} K_{n}\left(R_{F}\right)$ \\
\hline 4 & $s+t-3$ \\
5 & 0 \\
6 & $s+t-3$ \\
7 & 1 \\
\hline
\end{tabular}

Pr o of. Compare Theorem 1.1, Corollaries 2.10 and 2.11.

3.2. A method for two-primary calculations of $K_{2}$ for quadratic number rings. In this subsection we describe a method that in some favorable cases calculates the two-primary part of $K_{2}$ of quadratic number rings.

The narrow version of $K_{2}(F)$ is defined by a split short exact sequence

$$
0 \rightarrow K_{2}^{+}(F) \rightarrow K_{2}(F) \rightarrow \bigoplus_{\wp \text { real }} \mu_{2} \rightarrow 0
$$

induced by Hilbert symbols. Next one defines $K_{2}^{+}\left(\mathcal{O}_{F, S}\right):=K_{2}\left(\mathcal{O}_{F, S}\right) \cap$ $K_{2}^{+}(F)$.

The following result is part of Corollary 3.9 in [8].

Theorem 3.2 (Keune). For $F=\mathbb{Q}(\sqrt{d})$ we have

$$
\mathrm{rk}_{2} K_{2}^{+}\left(R_{F}\right)=\operatorname{rk}_{2} \mathrm{Pic}_{+}\left(R_{F}\right)+s-1 .
$$

One main reason for focusing on $K_{2}$ is that we have 4-rank formulas for $K_{2}$ of quadratic number fields. We continue to work with the quadratic number field $F=\mathbb{Q}(\sqrt{d})$ where $d$ is a squarefree integer. Let $\bar{F}=\mathbb{Q}(\sqrt{-d})$ with two-integers $R_{\bar{F}}$ and let $\delta=\mathrm{rk}_{2} \mathrm{Pic}_{+}\left(R_{F}\right)-\mathrm{rk}_{2} \mathrm{Pic}\left(R_{F}\right)$. It follows from Theorem 2.9 that $\delta$ equals 0 if $d \in M_{-1} \cup M_{-2}$ and 1 in all other cases. Various 4-rank formulas for $K_{2}$ are known (see [5] and [9]). We use the following results from [1].

Theorem 3.3 (Boldy). For $F=\mathbb{Q}(\sqrt{d})$ we have

$$
\operatorname{rk}_{4} K_{2}^{+}\left(R_{F}\right)=\operatorname{rk}_{4} \operatorname{Pic}_{+}\left(R_{\bar{F}}\right)+\bar{s}-1 .
$$

Theorem 3.4 (Boldy). For $F=\mathbb{Q}(\sqrt{d})$ we have

$$
\mathrm{rk}_{4} K_{2}\left(R_{F}\right)=\operatorname{rk}_{4} \mathrm{Pic}_{+}\left(R_{\bar{F}}\right)+\bar{s}+\delta-1 .
$$

Definition 3.5. Let $\mathrm{rk}_{n}$ denote the $n$-rank of $K_{2}\left(R_{F}\right)$ and $\mathrm{rk}_{n}^{+}$denote the $n$-rank of $K_{2}^{+}\left(R_{F}\right)$. Here $n$ is a prime power. We will only consider $n=2$ and $n=4$.

Let $p_{1}, \ldots, p_{\bar{t}}$ be the prime divisors of $\Delta_{\bar{F}}$ with the modification that $p_{\bar{t}}=2$ in case $2 \mid \Delta_{\bar{F}}$. Likewise we let $\bar{s}$ denote the number of dyadic primes 
in $\bar{F}$. The matrix

$$
M_{F}=\left[\begin{array}{cccc}
\left(\frac{d / p_{1}}{p_{1}}\right) & \left(\frac{p_{1}}{p_{2}}\right) & \cdots & \left(\frac{p_{1}}{p_{\bar{t}-1}}\right) \\
\left(\frac{p_{2}}{p_{1}}\right) & \left(\frac{d / p_{2}}{p_{2}}\right) & \cdots & \left(\frac{p_{2}}{p_{\bar{t}-1}}\right) \\
\vdots & \vdots & \ddots & \vdots \\
\left(\frac{p_{\bar{t}-1}}{p_{1}}\right) & \left(\frac{p_{\bar{t}-1}}{p_{2}}\right) & \cdots & \left(\frac{d / p_{\bar{t}-1}}{p_{\bar{t}-1}}\right) \\
\left(\frac{p_{\bar{t}}}{p_{1}}\right) & \left(\frac{p_{\bar{t}}}{p_{2}}\right) & \cdots & \left(\frac{p_{\bar{t}}}{p_{\bar{t}-1}}\right)
\end{array}\right]
$$

is described in [10], using ideas of Rédei. Here $\left(\frac{p}{q}\right)$ denotes the quadratic residue symbol of $p$ and $q$. We adopt the following conventions. The value in $M_{F}$ of $\left(\frac{p}{q}\right)=1$ is 0 , while the $M_{F}$ value of $\left(\frac{p}{q}\right)=-1$ is 1 . Let

$$
r_{2}=\operatorname{rk}_{2} \operatorname{Pic}_{+}\left(\mathcal{O}_{\bar{F}}\right)=\bar{t}-1 \text { and } r_{4}=\operatorname{rk}_{4} \operatorname{Pic}_{+}\left(\mathcal{O}_{\bar{F}}\right) .
$$

Then

$$
r_{4}=r_{2}-\operatorname{rank}_{\mathbb{F}_{2}}\left(M_{F}\right) .
$$

This fact motivates the definition of $M_{F}$.

From Theorems 3.3 and 3.4 we see that if we know the value of $\mathrm{rk}_{4}^{+}$, then we also know the value of $\mathrm{rk}_{4}$ since their difference is given by $\delta$. What we will do is to express $\mathrm{rk}_{2}^{+}$and $\mathrm{rk}_{4}^{+}$in terms of $r_{2}$ and $r_{4}$.

The first problem is to find $\mathrm{rk}_{4} \mathrm{Pic}_{+}\left(R_{\bar{F}}\right)$. As we saw in Subsection 2.3 there are cases where it is hard to find this number. We will run into trouble in the cases:

Situation 1: $[\wp]_{+} \in \operatorname{Pic}_{+}\left(\mathcal{O}_{\bar{F}}\right)^{4}$.

Situation $2:\left(\operatorname{ord}\left([\wp]_{+}\right) \equiv 0 \bmod 4\right.$ and $\left.[\wp]_{+} \in \operatorname{Pic}_{+}\left(\mathcal{O}_{\bar{F}}\right)^{4}\right)$ or $\left(\operatorname{ord}\left([\wp]_{+}\right)\right.$ $\equiv 2 \bmod 4)$.

Translating the Lemmas $2.15-2.17$ from $F$ to $\bar{F}$ yields

Corollary 3.6. We have $\operatorname{rk}_{4} \mathrm{Pic}_{+}\left(R_{\bar{F}}\right)=\operatorname{rk}_{4} \mathrm{Pic}_{+}\left(\mathcal{O}_{\bar{F}}\right)$ in the three cases:

1) $d \equiv 3,5 \bmod 8$,

2) $d \equiv 1,2,6 \bmod 8$ with the restriction $\left(d \notin M_{2}\right)$ or Situation 1 ,

3) $d \equiv 7 \bmod 8$ with the restriction

Situation 1 or $\left(d \notin M_{2}, \operatorname{ord}\left([\wp]_{+}\right) \equiv 2 \bmod 4\right)$

$$
\text { or }\left(d \notin M_{2},[\wp]_{+}^{2} \in \operatorname{Pic}_{+}\left(\mathcal{O}_{\bar{F}}\right)^{4}, \operatorname{ord}\left([\wp]_{+}\right) \equiv 0 \bmod 4\right) .
$$

In all other cases we have $\operatorname{rk}_{4} \mathrm{Pic}_{+}\left(\mathcal{O}_{\bar{F}}\right)-\operatorname{rk}_{4} \operatorname{Pic}_{+}\left(R_{\bar{F}}\right)=1$. 
Proposition 3.7. We have the following calculation of $\mathrm{rk}_{2}^{+}$and $\mathrm{rk}_{4}^{+}$:

\begin{tabular}{ccccc}
\hline$d \equiv \bmod 8$ & Case & with the condition & $\mathrm{rk}_{2}^{+}$ & $\mathrm{rk}_{4}^{+}$ \\
\hline 1 & 1.1 & $d \notin M_{2}$ & $r_{2}-1$ & $r_{4}$ \\
1 & 1.2 & $d \in M_{2}$ and Sit. 1 & $r_{2}$ & $r_{4}$ \\
1 & 1.2 & $d \in M_{2}$ and not Sit. 1 & $r_{2}$ & $r_{4}-1$ \\
3 & 2 & & $r_{2}$ & $r_{4}$ \\
5 & 3 & & $r_{2}-1$ & $r_{4}$ \\
2,6 & 4.1 & $d \notin M_{2}$ & $r_{2}-1$ & $r_{4}$ \\
2,6 & 4.2 & $d \in M_{2}$ and Sit. 1 & $r_{2}$ & $r_{4}$ \\
2,6 & 4.2 & $d \in M_{2}$ and not Sit. 1 & $r_{2}$ & $r_{4}-1$ \\
7 & 5.1 & $d \notin M_{2}$ and Sit. 1 & $r_{2}$ & $r_{4}+1$ \\
7 & 5.1 & $d \notin M_{2}$ and not Sit. 1 & $r_{2}$ & $r_{4}$ \\
7 & 5.2 & $d \in M_{2}$ and Sit. 1 & $r_{2}+1$ & $r_{4}+1$ \\
7 & 5.2 & $d \in M_{2}$ and not Sit. 1 & $r_{2}+1$ & $r_{4}$ \\
\hline
\end{tabular}

with the additional remark:

If $d \equiv 7 \bmod 8$ and $d \in M_{2}$, then $r_{4}=0$ implies $\mathrm{rk}_{4}^{+}=1$.

Pr o of. CASE 1.1: $d \equiv 1 \bmod 8$ and $d \notin M_{2}$. Then $\operatorname{rk}_{2} \mathrm{Pic}_{+}\left(\mathcal{O}_{F}\right)=r_{2}-1$, $\operatorname{rk}_{2} \mathrm{Pic}_{+}\left(R_{F}\right)=r_{2}-2$ and $\operatorname{rk}_{4} \mathrm{Pic}_{+}\left(R_{\bar{F}}\right)=r_{4}$. Further $s=2$ and $\bar{s}=1$. Hence $\mathrm{rk}_{2}^{+}=r_{2}-1$ and $\mathrm{rk}_{4}^{+}=r_{4}$.

CASE 1.2: $d \equiv 1 \bmod 8, d \in M_{2}$. We get that $\operatorname{rk}_{2} \mathrm{Pic}_{+}\left(\mathcal{O}_{F}\right)=r_{2}-1$, $\mathrm{rk}_{2} \mathrm{Pic}_{+}\left(R_{F}\right)=r_{2}-1, \mathrm{rk}_{4} \mathrm{Pic}_{+}\left(R_{\bar{F}}\right)=r_{4}$ in Situation 1 and $\mathrm{rk}_{4} \mathrm{Pic}_{+}\left(R_{\bar{F}}\right)=$ $r_{4}-1$ in all other cases. Further $s=2$ and $\bar{s}=1$. Hence $\mathrm{rk}_{2}^{+}=r_{2}, \mathrm{rk}_{4}^{+}=r_{4}$ if Situation 1 holds and $\mathrm{rk}_{4}^{+}=r_{4}-1$ otherwise.

CASE 2: $d \equiv 3 \bmod 8$. We derive that $\operatorname{rk}_{2} \mathrm{Pic}_{+}\left(\mathcal{O}_{F}\right)=r_{2}+1, \operatorname{rk}_{2} \mathrm{Pic}_{+}\left(R_{F}\right)$ $=r_{2}$ since $d \notin M_{2}$, and also $\operatorname{rk}_{4} \mathrm{Pic}_{+}\left(R_{\bar{F}}\right)=r_{4}$. Further $s=\bar{s}=1$. We find that $\mathrm{rk}_{2}^{+}=r_{2}$ and $\mathrm{rk}_{4}^{+}=r_{4}$.

CASE 3: $d \equiv 5 \bmod 8$. We get $\operatorname{rk}_{2} \mathrm{Pic}_{+}\left(\mathcal{O}_{F}\right)=r_{2}-1, \operatorname{rk}_{2} \mathrm{Pic}_{+}\left(R_{F}\right)=$ $r_{2}-1$ and $\operatorname{rk}_{4} \mathrm{Pic}_{+}\left(R_{\bar{F}}\right)=r_{4}$. Further $s=\bar{s}=1$. This time we find $\mathrm{rk}_{2}^{+}=r_{2}$ and $\mathrm{rk}_{4}^{+}=r_{4}$.

CASE 4.1: $d \equiv 2,6 \bmod 8, d \notin M_{2}$. We get $\operatorname{rk}_{2} \mathrm{Pic}_{+}\left(\mathcal{O}_{F}\right)=r_{2}-1$, $\operatorname{rk}_{2} \mathrm{Pic}_{+}\left(R_{F}\right)=r_{2}-1, \operatorname{rk}_{4} \mathrm{Pic}_{+}\left(R_{\bar{F}}\right)=r_{4}$. Further $s=\bar{s}=1$. Hence $\operatorname{rk}_{2}^{+}=$ $r_{2}-1$ and $\mathrm{rk}_{4}^{+}=r_{4}$.

CASE 4.2: $d \equiv 2,6 \bmod 8, d \in M_{2}$. Now $\operatorname{rk}_{2} \mathrm{Pic}_{+}\left(\mathcal{O}_{F}\right)=\operatorname{rk}_{2} \mathrm{Pic}_{+}\left(R_{F}\right)=$ $r_{2}, \operatorname{rk}_{4} \mathrm{Pic}_{+}\left(R_{\bar{F}}\right)=r_{4}$ if Situation 1 holds and $\operatorname{rk}_{4} \mathrm{Pic}_{+}\left(R_{\bar{F}}\right)=r_{4}-1$ otherwise. Again $s=\bar{s}=1$, so $\mathrm{rk}_{2}^{+}=r_{2}, \mathrm{rk}_{4}^{+}=r_{4}$ if Situation 1 holds and $\mathrm{rk}_{4}^{+}=r_{4}-1$ otherwise.

CASE 5.1: $d \equiv 7 \bmod 8, d \notin M_{2}$. We get $\operatorname{rk}_{2} \mathrm{Pic}_{+}\left(\mathcal{O}_{F}\right)=r_{2}+1$, $\operatorname{rk}_{2} \mathrm{Pic}_{+}\left(R_{F}\right)=r_{2}, \operatorname{rk}_{4} \mathrm{Pic}_{+}\left(R_{F}\right)=r_{4}$ if Situation 1 holds and $\operatorname{rk}_{4} \mathrm{Pic}_{+}\left(R_{\bar{F}}\right)$ $=r_{4}-1$ otherwise. Again $s=1$ and $\bar{s}=2$. Hence $\mathrm{rk}_{2}^{+}=r_{2}, \mathrm{rk}_{4}^{+}=r_{4}+1$ if Situation 1 holds and $\mathrm{rk}_{4}^{+}=r_{4}$ otherwise. 
CASE 5.2: $d \equiv 7 \bmod 8, d \in M_{2}$. We get $\operatorname{rk}_{2} \mathrm{Pic}_{+}\left(\mathcal{O}_{F}\right)=\operatorname{rk}_{2} \mathrm{Pic}_{+}\left(R_{F}\right)=$ $r_{2}+1, \operatorname{rk}_{4} \mathrm{Pic}_{+}\left(R_{\bar{F}}\right)=r_{4}$ in Situation 1 and $\operatorname{rk}_{4} \mathrm{Pic}_{+}\left(R_{\bar{F}}\right)=r_{4}-1$ otherwise. Trivially $s=1$ and $\bar{s}=2$. So $\mathrm{rk}_{2}^{+}=r_{2}+1, \mathrm{rk}_{4}^{+}=r_{4}+1$ if Situation 1 holds and $\mathrm{rk}_{4}^{+}=r_{4}$ otherwise.

Now for the additional remark. When $r_{4}=0$ we claim that $[\wp]_{+} \in$ $\mathrm{Pic}_{+}\left(\mathcal{O}_{\bar{F}}\right)^{4}$, i.e., we are in Situation 1. The condition on $d$ implies $[\wp]_{+} \in$ $\mathrm{Pic}_{+}\left(\mathcal{O}_{\bar{F}}\right)^{2}$ from Lemmas 2.5 and 2.7. The assumption $r_{4}=0$ implies $\mathrm{Pic}_{+}\left(\mathcal{O}_{\bar{F}}\right)^{2}=\mathrm{Pic}_{+}\left(\mathcal{O}_{\bar{F}}\right)^{4}$, i.e., $[\wp]_{+}$is a fourth power in $\mathrm{Pic}_{+}\left(\mathcal{O}_{\bar{F}}\right)$.

Now an outline of the method that we use for the calculations. Proposition 3.7 calculates $\mathrm{rk}_{2}^{+}$and $\mathrm{rk}_{4}^{+}$in terms of $r_{2}$ and $r_{4}$, respectively. It is trivial to find $r_{2}$, while the matrix $M_{F}$ takes care of $r_{4}$. In this way we automatically determine $\mathrm{rk}_{4}$ from $\delta$. The matrix $M_{F}$ will play the vital part in the calculations. Using this method with ideas going all the way back to Rédei we have a quite powerful machine in hand that provides lots of examples. This will be discussed in the next subsection.

3.3. Examples of $K_{2}$ of quadratic number rings. This subsection consists of examples. We will heavily use the formulas obtained in the previous subsection and the calculations will usually be based on the matrix $M_{F}$. Whenever we write the word matrix we refer to $M_{F}$. First we make an elementary observation.

EXAMPLE 3.8. Let $F$ be a real quadratic number field and suppose that $\mathrm{rk}_{4}^{+}=0$. Then

$$
K_{2}\left(R_{F}\right)\{2\} \cong(\mathbb{Z} / 2)^{\mathrm{rk}_{2}-\mathrm{rk}_{4}} \oplus(\mathbb{Z} / 4)^{\mathrm{rk}_{4}} .
$$

Pr o of. Recall that $\mathrm{rk}_{2}+\mathrm{rk}_{4}=\mathrm{rk}_{2}^{+}+\mathrm{rk}_{4}^{+}+2$ from the 2-rank and 4-rank formulas. From the exact sequence $0 \rightarrow K_{2}^{+}\left(R_{F}\right) \rightarrow K_{2}\left(R_{F}\right) \rightarrow \mu_{2} \oplus \mu_{2} \rightarrow 0$ we find that $\mathrm{rk}_{2}+\mathrm{rk}_{4}+\ldots=2+\mathrm{rk}_{2}^{+}+\mathrm{rk}_{4}^{+}+\ldots$, i.e., $\mathrm{rk}_{8}+\mathrm{rk}_{16}+\ldots=$ $\mathrm{rk}_{8}^{+}+\mathrm{rk}_{16}^{+}+\ldots$ But the assumption is that $\mathrm{rk}_{4}^{+}=0$.

Before we begin with the examples we ought to recall the following. For $d<0$ we clearly have $\mathrm{rk}_{2}=\mathrm{rk}_{2}^{+}$and $\mathrm{rk}_{4}=\mathrm{rk}_{4}^{+}$since the narrow and the usual $K_{2}$ are equal. For $d$ positive we have $\mathrm{rk}_{2}=\mathrm{rk}_{2}^{+}+2-\delta$ and $\mathrm{rk}_{4}=$ $\mathrm{rk}_{4}^{+}+\delta$. Again we emphasize that $\delta=0$ if $d \in M_{-1} \cup M_{-2}$, and 1 in all other cases. All the $p, q$ and $r$ 's occurring in the examples are prime numbers. In the calculations we will refer to the various cases occurring in Proposition 3.7. Let us begin with an easy example.

Example 3.9. Let $F=\mathbb{Q}(\sqrt{-p})$. Then

for some $n \geq 2$.

$$
K_{2}\left(R_{F}\right)\{2\} \cong \begin{cases}\mathbb{Z} / 2^{n} & \text { for } p \equiv 1 \bmod 8 \\ \mathbb{Z} / 2 & \text { for } p \equiv 7 \bmod 8 \\ 0 & \text { otherwise },\end{cases}
$$


Pr o of. We divide the calculation into cases according to the congruence class of $p$ modulo 8 .

1) $p \equiv 1 \bmod 8$. Now $d \equiv 7 \bmod 8, d \in M_{2}$, so we are in Case 5.2. From $d \equiv 3 \bmod 4$ we find that $\Delta=p$, i.e., $\bar{t}=1, r_{2}=r_{4}=0$. Thus we are in Situation 1 and $\mathrm{rk}_{2}=\mathrm{rk}_{4}=1$.

2) $p \equiv 3 \bmod 8$. We have $d \equiv 5 \bmod 8$, i.e., Case 3. From $d \equiv 1 \bmod 4$ we find that $\Delta=4 p$, i.e., $\bar{t}=2, r_{2}=1$ and hence $\mathrm{rk}_{2}=0$.

3) $p \equiv 5 \bmod 8$. We have $d \equiv 3 \bmod 8, d \notin M_{2}$, i.e., Case 2. From $d \equiv 3 \bmod 4$ we find that $\Delta=p$, i.e., $\bar{t}=1, r_{2}=0$ and hence $\mathrm{rk}_{2}=0$.

4) $p \equiv 7 \bmod 8$. We have $d \equiv 1 \bmod 8, d \in M_{2}$, i.e., Case 1.2. From $d \equiv 1 \bmod 4$ we find that $\Delta=4 p$, i.e., $\bar{t}=2, r_{2}=1$. The matrix is

$$
\left[\begin{array}{c}
\left(\frac{-d / p}{p}\right) \\
\left(\frac{2}{p}\right)
\end{array}\right]=\left[\begin{array}{c}
\left(\frac{-1}{p}\right) \\
\left(\frac{2}{p}\right)
\end{array}\right] \sim\left[\begin{array}{l}
1 \\
0
\end{array}\right]
$$

and hence $r_{4}=r_{2}-1=0$, i.e., $\mathrm{rk}_{2}-1=\mathrm{rk}_{4}=0$.

REMARK 3.10. One can prove that $n=2$ in the case $p \equiv 9 \bmod 16$. The situation is far more complicated if $p \equiv 1 \bmod 16$, because in this case $n \geq 3$. As an example we mention that $n=3$ for $p=17$. All these claims follow from unpublished work of Qin; see also [4] for similar computations.

Next we consider $F=\mathbb{Q}\left(\sqrt{-p_{1} \ldots p_{2 m+1} q_{1} \ldots q_{2 n+1}}\right)$ with $p_{i} \equiv 7 \bmod 8$ and $q_{i} \equiv 5 \bmod 8$. From the residue classes of the $p_{i}$ 's and $q_{i}$ 's modulo eight we have $\left(\frac{p_{i}}{p_{j}}\right)=\left(\frac{p_{j}}{p_{i}}\right)$. We are in Case 3 , and we easily find $\mathrm{rk}_{2}=2(m+n)+1$ from Theorem 3.1. Let $\widehat{p}_{i}$ denote the product $p_{1} \ldots p_{i-1} p_{i+1} \ldots q_{2 n-1}$ and likewise for $\widehat{q}_{i}$. The matrix is

$$
M_{F}=\left[\begin{array}{cccc}
-\left(\frac{\widehat{p}_{1}}{p_{1}}\right) & \left(\frac{p_{1}}{p_{2}}\right) & \cdots & \left(\frac{p_{1}}{q_{2 n-1}}\right) \\
\left(\frac{p_{2}}{p_{1}}\right) & -\left(\frac{\widehat{p}_{2}}{p_{2}}\right) & \cdots & \left(\frac{p_{2}}{q_{2 n-1}}\right) \\
\vdots & \vdots & \ddots & \vdots \\
\left(\frac{q_{2 n+1}}{p_{1}}\right) & \left(\frac{q_{2 n+1}}{p_{2}}\right) & \cdots & \left(\frac{\widehat{q}_{2 n+1}}{q_{2 n+1}}\right) \\
\left(\frac{2}{p_{1}}\right) & \left(\frac{2}{p_{2}}\right) & \cdots & \left(\frac{2}{q_{2 n+1}}\right)
\end{array}\right] .
$$

We make some observations on $M_{F}$. First assume that all the quadratic residue symbols are 1 . Then the entries in the first $2 m+1$ columns are all zero except $e_{i i}=1$ for $1 \leq i \leq 2 m+1$. Next consider the remaining $2 n+1$ columns. All the entries are zero except the last row where all the entries are 1 . Thus the $\mathbb{F}_{2}$-rank of $M_{F}$ is $2 m+2$ and $\mathrm{rk}_{4}=2 n$. Hence we record

EXAMPLE 3.11. Let $F=\mathbb{Q}\left(\sqrt{-p_{1} \ldots p_{2 m+1} q_{1} \ldots q_{2 n+1}}\right)$ with $p_{i} \equiv 7 \bmod 8$ and $q_{i} \equiv 5 \bmod 8$. If all the quadratic symbols we can make from the $p_{i}$ 's 
and $q_{i}$ 's are 1 , then

$$
K_{2}\left(R_{F}\right)\{2\} \cong(\mathbb{Z} / 2)^{2 m+1} \oplus\left(\mathbb{Z} / 2^{a_{1}}\right) \oplus \ldots \oplus\left(\mathbb{Z} / 2^{a_{2 n}}\right)
$$

for some $a_{i} \geq 2$.

Next consider the following slight modification of the last example. We adjoin one more $p_{i}$, say $p_{2 m+2}$, such that there are an even number of $p_{i}$ 's. This change of input brings us over to Case 2 . The 2 -rank rk $\mathrm{rk}_{2}$ equals $2(m+$ $n+1)$, and

$$
M_{F}=\left[\begin{array}{cccc}
-\left(\frac{\widehat{p}_{1}}{p_{1}}\right) & \left(\frac{p_{1}}{p_{2}}\right) & \cdots & \left(\frac{p_{1}}{q_{2 n-1}}\right) \\
\left(\frac{p_{2}}{p_{1}}\right) & -\left(\frac{\widehat{p}_{2}}{p_{2}}\right) & \cdots & \left(\frac{p_{2}}{q_{2 n-1}}\right) \\
\vdots & \vdots & \ddots & \vdots \\
\left(\frac{q_{2 n}}{p_{1}}\right) & \left(\frac{q_{2 n}}{p_{2}}\right) & \cdots & \left(\frac{\widehat{q}_{2 n}}{q_{2 n}}\right) \\
\left(\frac{q_{2 n+1}}{p_{1}}\right) & \left(\frac{q_{2 n+1}}{p_{2}}\right) & \cdots & \left(\frac{q_{2 n+1}}{q_{2 n}}\right)
\end{array}\right] .
$$

If all the quadratic residue symbols are 1 , then $e_{i i}=1$ for $1 \leq i \leq 2 m+2$ while all the other entries are zero. Consequently,

$$
K_{2}\left(R_{F}\right)\{2\} \cong(\mathbb{Z} / 2)^{2 m+2} \oplus\left(\mathbb{Z} / 2^{a_{1}}\right) \oplus \ldots \oplus\left(\mathbb{Z} / 2^{a_{2 n}}\right)
$$

for some $a_{i} \geq 2$. Suppose the only nontrivial quadratic symbols are $\left(\frac{p_{1}}{p_{2}}\right)=$ $\ldots=\left(\frac{p_{i}}{p_{i+1}}\right)=-1$. The number $\mathrm{rk}_{4}$ equals $2 n+1$ if $i$ is odd, as one easily checks by looking at $M_{F}$. However, $\mathrm{rk}_{4}$ will be $2 n+1$ if $i$ is even.

EXAMPLE 3.12. Let $F=\mathbb{Q}\left(\sqrt{-p_{1} \ldots p_{2 m+2} q_{1} \ldots q_{2 n+1}}\right)$ with $p_{i} \equiv 7 \bmod 8$ and $q_{i} \equiv 5 \bmod 8$. If the only nontrivial quadratic symbols are $\left(\frac{p_{1}}{p_{2}}\right)=\ldots=$ $\left(\frac{p_{i}}{p_{i+1}}\right)=-1$ where $i$ is even, then

$$
K_{2}\left(R_{F}\right)\{2\} \cong(\mathbb{Z} / 2)^{2 m+1} \oplus\left(\mathbb{Z} / 2^{a_{1}}\right) \oplus \ldots \oplus\left(\mathbb{Z} / 2^{a_{2 n+1}}\right)
$$

for some $a_{i} \geq 2$.

Next consider the real quadratic number field $F=\mathbb{Q}\left(\sqrt{p_{1} \ldots p_{2 n+1}}\right)$ for some $p_{i} \equiv 3 \bmod 8$. One checks that $\mathrm{rk}_{2}=2 n+2, \mathrm{rk}_{2}^{+}=2 n$ and $\left(\frac{p_{i}}{p_{j}}\right)=-\left(\frac{p_{j}}{p_{i}}\right)$. The matrix is

$$
M_{F}=\left[\begin{array}{ccc}
\left(\frac{\widehat{p}_{1}}{p_{1}}\right) & \cdots & \left(\frac{p_{1}}{p_{2 n}}\right) \\
\left(\frac{p_{2}}{p_{1}}\right) & \cdots & \left(\frac{p_{2}}{p_{2 n}}\right) \\
\vdots & \ddots & \vdots \\
\left(\frac{p_{2 n-1}}{p_{1}}\right) & \cdots & \left(\frac{\widehat{p}_{2 n-1}}{p_{2 n-1}}\right) \\
\left(\frac{p_{2 n}}{p_{1}}\right) & \cdots & \left(\frac{p_{2 n}}{p_{2 n-1}}\right)
\end{array}\right] .
$$


If all the possible quadratic residue symbols we can make out of the $p_{i}$ 's are trivial, then $\mathrm{rk}_{4}=\mathrm{rk}_{2}$. By looking at the matrix one finds that if $\left(\frac{p_{1}}{p_{2}}\right), \ldots,\left(\frac{p_{i-1}}{p_{i}}\right)$ are the only quadratic residue symbols equal to -1 , then $\mathrm{rk}_{4}=\mathrm{rk}_{4}^{+}=2 n-i+1$. Hence

EXAMPLE 3.13. Let $F=\mathbb{Q}\left(\sqrt{p_{1} \cdots p_{2 n+1}}\right)$ with $p_{i} \equiv 3 \bmod 8$. If $\left(\frac{p_{1}}{p_{2}}\right)=$ $\ldots=\left(\frac{p_{i-1}}{p_{i}}\right)$ are the only quadratic residue symbols equal to -1 , then

$$
K_{2}^{+}\left(R_{F}\right)\{2\} \cong(\mathbb{Z} / 2)^{i+1} \oplus\left(\mathbb{Z} / 2^{a_{1}}\right) \oplus \ldots \oplus\left(\mathbb{Z} / 2^{a_{2 n-i-1}}\right)
$$

and

$$
K_{2}\left(R_{F}\right)\{2\} \cong(\mathbb{Z} / 2)^{i+3} \oplus\left(\mathbb{Z} / 2^{b_{1}}\right) \oplus \ldots \oplus\left(\mathbb{Z} / 2^{b_{2 n-i-1}}\right)
$$

for some $a_{j}, b_{j} \geq 2$.

We end the section with two more concrete examples.

EXAmple 3.14. For $F=\mathbb{Q}(\sqrt{-p q r})$ with $p, q \equiv 7 \bmod 8, r \equiv 5 \bmod 8$ and $\left(\frac{p}{r}\right)=-1$

$$
K_{2}\left(R_{F}\right)\{2\} \cong \mathbb{Z} / 2 \oplus \mathbb{Z} / 2
$$

Proof. We are in Case 2. One finds that $\mathrm{rk}_{2}=2$ and $\mathrm{rk}_{4}=r_{4}$. The matrix is:

$$
\left[\begin{array}{ll}
1 & 1 \\
0 & 1 \\
1 & 0
\end{array}\right], \quad\left[\begin{array}{ll}
1 & 0 \\
0 & 1 \\
1 & 1
\end{array}\right], \quad\left[\begin{array}{ll}
0 & 1 \\
1 & 0 \\
1 & 0
\end{array}\right] \text { or }\left[\begin{array}{ll}
0 & 0 \\
1 & 0 \\
1 & 1
\end{array}\right] \text {. }
$$

Hence $r_{4}=2-\operatorname{rk}_{\mathbb{F}_{2}}\left(M_{F}\right)=0$.

ExAmPle 3.15. Let $F=\mathbb{Q}(\sqrt{2 p q r})$ with $p \equiv 1 \bmod 8, q \equiv 3 \bmod 8$ and $r \equiv 5 \bmod 8$. Assume that $-\left(\frac{q}{p}\right)=\left(\frac{r}{p}\right)=1$. Then

$$
K_{2}^{+}\left(R_{F}\right)\{2\} \cong \mathbb{Z} / 2 \oplus \mathbb{Z} / 2
$$

and

$$
K_{2}\left(R_{F}\right)\{2\} \cong \mathbb{Z} / 2 \oplus \mathbb{Z} / 2 \oplus \mathbb{Z} / 2^{n}
$$

for some $n \geq 2$.

Proof. We are in Case 4.1, $\mathrm{rk}_{2}=2$ and $\mathrm{rk}_{4}=r_{4}$. The matrix is

$$
\left[\begin{array}{ccc}
\left(\frac{2 q r}{p}\right) & \left(\frac{p}{q}\right) & \left(\frac{p}{r}\right) \\
\left(\frac{q}{p}\right) & \left(\frac{2 p r}{q}\right) & \left(\frac{q}{r}\right) \\
\left(\frac{r}{p}\right) & \left(\frac{r}{q}\right) & \left(\frac{2 p q}{r}\right) \\
\left(\frac{2}{p}\right) & \left(\frac{2}{q}\right) & \left(\frac{2}{r}\right)
\end{array}\right]=\left[\begin{array}{ccc}
1 & 1 & 0 \\
1 & \left(\frac{r}{q}\right) & \left(\frac{r}{q}\right) \\
0 & \left(\frac{r}{q}\right) & -\left(\frac{r}{q}\right) \\
0 & 1 & 1
\end{array}\right] \sim \ldots \sim\left[\begin{array}{ccc}
1 & 0 & 0 \\
0 & 1 & 0 \\
0 & 0 & 1 \\
0 & 0 & 0
\end{array}\right]
$$

and hence $\mathrm{rk}_{4}^{+}=0$. Since $d \notin M_{-1} \cup M_{-2}$ we find that $\delta=1, \mathrm{rk}_{2}=3$ and $\mathrm{rk}_{4}=1$. 
3.4. Lifting the $K_{2}$ calculations to higher K-groups. The basis for lifting the previous $K_{2}$ calculations to higher algebraic K-groups is

Theorem 3.16 (Rognes-Weibel). Let $F$ be a totally imaginary number field. Then

$$
K_{2 n}\left(R_{F}\right)\{2\} \cong H_{\text {ét }}^{2}\left(R ; \mathbb{Z}_{2}(n+1)\right) .
$$

For a real number field $F$,

$$
K_{8 n+2}\left(R_{F}\right)\{2\} \cong H_{\text {ét }}^{2}\left(R ; \mathbb{Z}_{2}(4 n+2)\right) .
$$

Let $e$ denote the exponent of $\left(\mathbb{Z} / 2^{\nu}\right)^{*}$. The motivation for introducing $e$ is the isomorphism $\mathbb{Z} / 2^{\nu}(i) \cong \mathbb{Z} / 2^{\nu}(e+i)$ of sheaves for the étale topology. The exact sequence

$$
0 \rightarrow \mathbb{Z}_{2}(i) \stackrel{2^{\nu}}{\rightarrow} \mathbb{Z}_{2}(i) \rightarrow \mathbb{Z} / 2^{\nu}(i) \rightarrow 0
$$

of coefficient sheaves induces the horizontal exact sequence diagram:

$$
\begin{aligned}
& 0 \rightarrow H_{\text {êt }}^{2}\left(R_{F} ; \mathbb{Z}_{2}(2)\right) / 2^{\nu} \rightarrow H_{\text {êt }}^{2}\left(R_{F} ; \mathbb{Z} / 2^{\nu}(2)\right) \rightarrow 2^{\nu} H_{\text {êt }}^{3}\left(R_{F} ; \mathbb{Z}_{2}(2)\right) \rightarrow 0
\end{aligned}
$$

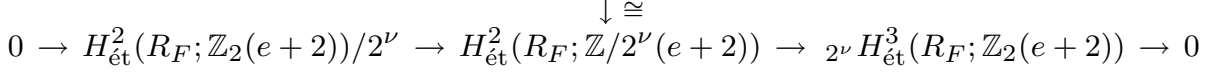

Lemma 3.17. Let $i \neq 0,1$. Then

$$
H_{\text {èt }}^{3}\left(R_{F} ; \mathbb{Z}_{2}(i)\right) \cong \begin{cases}(\mathbb{Z} / 2)^{r_{1}} & \text { for } i \text { odd }, \\ 0 & \text { for } i \text { even } .\end{cases}
$$

Pro of. Combine Corollary 4.4, Propositions 2.4 and 4.6 of [15].

Combining the diagram, Theorem 3.16 and Lemma 3.17 yields

TheOREM 3.18. Let $e$ be the exponent of $\left(\mathbb{Z} / 2^{\nu}\right)^{*}$ where $\nu \geq 2$ if $F$ is a real number field. Recall that $e=2^{\nu-1}$ for $1 \leq \nu \leq 2$, and $e=2^{\nu-2}$ for $\nu \geq 3$. We have the isomorphism

$$
K_{2}\left(R_{F}\right) / 2^{\nu} \cong H_{\text {ét }}^{2}\left(R_{F} ; \mathbb{Z}_{2}(e+2)\right) / 2^{\nu} .
$$

In particular if $F$ is totally imaginary, or if $F$ is real and $e \equiv 0 \bmod 4$, then

$$
K_{2}\left(R_{F}\right) / 2^{\nu} \cong K_{2 e+2}\left(R_{F}\right) / 2^{\nu} .
$$

REMARK 3.19. This result lifts the $K_{2}$ calculations to higher K-groups. This illustrates in yet another way how important and interesting $K_{2}$ is!

We illustrate this result using the field $F$ from Example 3.14. Recall that $K_{2}\left(R_{F}\right)\{2\} \cong(\mathbb{Z} / 2)^{2}$. Thus $K_{2}\left(R_{F}\right)\{2\} \cong K_{2}\left(R_{F}\right) / 2$ and $K_{2}\left(R_{F}\right)\{2\} \cong$ $K_{2 e+2}\left(R_{F}\right) / 2^{\nu}$.

\section{References}

[1] M. C. Boldy, The 2-primary component of the tame kernel of quadratic number fields, Ph.D. thesis, Catholic University of Nijmegen, 1991. 
[2] A. Borel, Cohomologie réelle stable des groupes S-arithmétiques classiques, C. R. Acad. Sci. Paris 7 (1974), 235-272.

[3] J. Browkin and H. Gangl, Table of tame and wild kernels of quadratic imaginary number fields of discriminants $>-5000$ (conjectural values), Math. Comp., to appear.

[4] J. Browkin and A. Schinzel, On Sylow 2-subgroups of $\mathrm{K}_{2} \mathrm{O}_{F}$ for quadratic number fields F, J. Reine Angew. Math. 331 (1982), 104-113.

[5] P. E. Conner and J. Hurrelbrink, The 4-rank of $K_{2}(\mathcal{O})$, Canad. J. Math. 41 (1989), 932-960.

[6] A. Fröhlich and R. Taylor, Algebraic Number Theory, Cambridge Stud. Adv. Math. 27, Cambridge Univ. Press, 1993.

[7] M. Ishida, The Genus Fields of Algebraic Number Fields, Lecture Notes in Math. 555, Springer, 1976.

[8] F. Keune, On the structure of the $K_{2}$ of ring of integers in a number field, KTheory 2 (1989), 625-645.

[9] M. Kolster, The structure of the 2-Sylow subgroup of $K_{2}(\mathcal{O}), I$, Comment. Math. Helv. 61 (1986), 376-388.

[10] P. Morton, On Redei's theory of the Pell equation, J. Reine Angew. Math. 307/308 (1978), 373-398.

[11] J. Neukirch, Class Field Theory, Grundlehren Math. Wiss. 280, Springer, 1986.

[12] H. Qin, The 2-Sylow subgroups of the tame kernel of imaginary quadratic fields, Acta Arith. 69 (1995), 153-169.

[13] -, The 4-rank of $K_{2} O_{F}$ for real quadratic fields $F$, ibid. 72 (1995), 323-333.

[14] D. Quillen, Finite Generation of the Groups $K_{i}$ of Rings of Algebraic Integers, Lectures Notes in Math. 341, Springer, 1973, 179-198.

[15] J. Rognes and C. Weibel, Two-primary algebraic K-theory of rings of integers in number fields, preprint, 1997; http://www.math.uiuc.edu/K-theory/0220/.

[16] J. Tate, Relations between $K_{2}$ and Galois cohomology, Invent. Math. 36 (1976), $257-274$.

[17] A. Vazzana, On the 2-primary part of $K_{2}$ of rings of integers in certain quadratic number fields, Acta Arith. 80 (1997), 225-235.

[18] - Elementary abelian 2-primary parts of $K_{2} \mathrm{O}$ and related graphs in certain quadratic number fields, ibid. 81 (1997), 253-264.

Department of Mathematics

University of Utrecht

Utrecht, The Netherlands

E-mail: crainic@math.ruu.nl
Department of Mathematics

University of Oslo

Oslo, Norway

E-mail: paularne@math.uio.no

Received on 21.10.1997

and in revised form on 27.2.1998 